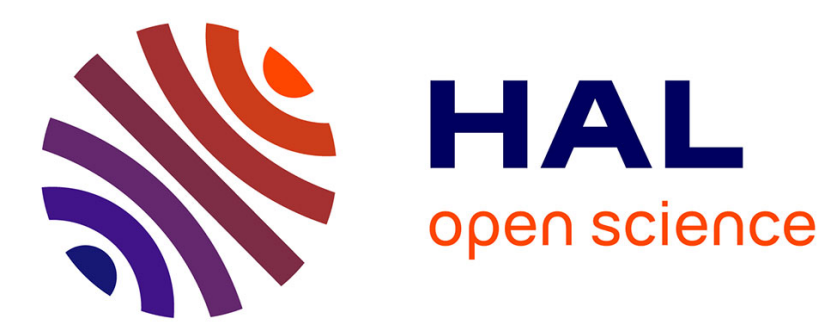

\title{
Analytical estimation of dynamic apertures limited by the wigglers in storage rings
}

\author{
J. Gao
}

\section{To cite this version:}

J. Gao. Analytical estimation of dynamic apertures limited by the wigglers in storage rings. Nuclear Instruments and Methods in Physics Research Section A: Accelerators, Spectrometers, Detectors and Associated Equipment, 2004, 516, pp.243-248. in2p3-00020253

HAL Id: in2p3-00020253

https://hal.in2p3.fr/in2p3-00020253

Submitted on 16 Jan 2004

HAL is a multi-disciplinary open access archive for the deposit and dissemination of scientific research documents, whether they are published or not. The documents may come from teaching and research institutions in France or abroad, or from public or private research centers.
L'archive ouverte pluridisciplinaire HAL, est destinée au dépôt et à la diffusion de documents scientifiques de niveau recherche, publiés ou non, émanant des établissements d'enseignement et de recherche français ou étrangers, des laboratoires publics ou privés. 


\title{
Analytical Estimation of Dynamic Apertures Limited by the Wigglers in Storage Rings
}

\author{
J. Gao \\ Laboratoire de L'Accélérateur Linéaire \\ IN2P3-CNRS et Université de Paris-Sud \\ B.P. 34, 91898 Orsay cedex, France
}

\begin{abstract}
By applying the general dynamic aperture formulae for the multipoles in a storage ring developed in ref. [1] (J. Gao, Nucl. Instr. and Methods A451 (2000), p. 545), in this paper, we give the analytical formulae for the dynamic apertures limited by the wigglers in storage rings. 1
\end{abstract}

\section{Introduction}

Wigller as an insertion device finds many applications in damping rings [2], synchrotron radiation facilities [3] [4], and storage ring colliders [5]. Intrinsically, as a nonlinear device, together with the perturbations to the linear optics it brings additional limitations to the general performance of the machines, such as reducing dynamic apertures. In this paper, we will estimate in an analytical way the dynamic apertures limited by wigglers. Firstly, in section 2 , we make a brief review of the beam dynamics inside a wiggler, and secondly, in sections 3 a wiggler is inserted into a storage ring as a perturbation. By applying the general dynamic aperture formulae of multipoles in a storage ring developed in ref. [1], in section 4 we derived analytical formulae of the wiggler limited dynamic aperture. Finally, in section 5 some numerical examples will be given.

\footnotetext{
${ }^{1}$ TESLA Report 2003-15
} 


\section{Particle's motion inside a wiggler}

Considering a wiggler of sinusoidal magnetic field variation, one can express the wiggler's magnetic fields, which satisfies Maxwell equations, as follows:

$$
\begin{gathered}
B_{x}=\frac{k_{x}}{k_{y}} B_{0} \sinh \left(k_{x} x\right) \sinh \left(k_{y} y\right) \cos (k s) \\
B_{y}=B_{0} \cosh \left(k_{x} x\right) \cosh \left(k_{y} y\right) \cos (k s) \\
B_{z}=-\frac{k}{k_{y}} B_{0} \cosh \left(k_{x} x\right) \sinh \left(k_{y} y\right) \sin (k s)
\end{gathered}
$$

with

$$
k_{x}^{2}+k_{y}^{2}=k^{2}=\left(\frac{2 \pi}{\lambda_{w}}\right)^{2}
$$

where $B_{0}$ is the peak sinusoidal wiggler magnetic field, $\lambda_{w}$ is the period length of the wiggler, and $x, y, s$ represent horizontal, vertical, and beam moving directions, respectively.

The Hamiltonian describing particle's motion can be written as [3]:

$$
H_{w}=\frac{1}{2}\left(p_{z}^{2}+\left(p_{x}-A_{x} \sin (k s)\right)^{2}+\left(p_{y}-A_{y} \sin (k s)\right)^{2}\right)
$$

where

$$
\begin{aligned}
& A_{x}=\frac{1}{\rho_{w} k} \cosh \left(k_{x} x\right) \cosh \left(k_{y} y\right) \\
& A_{x}=-\frac{k_{x}}{k_{y}} \frac{\sinh \left(k_{x} x\right) \sinh \left(k_{y} y\right)}{\rho_{w} k}
\end{aligned}
$$

and $\rho_{w}$ is the radius of curvature of the wiggler peak magnetic field $B_{0}$, and $\rho_{w}=E_{0} / e c B_{0}$ with $E_{0}$ being the electron energy. After making a canonical transformation to betatron variables, averaging the Hamiltonian over one period of wiggler, and expanding the hyperbolic functions to the fourth order in $x$ and $y$, one gets:

$$
\begin{aligned}
\mathcal{H}_{w}=\frac{1}{2}\left(p_{x}^{2}+p_{y}^{2}\right)+ & \frac{1}{4 k^{2} \rho_{w}^{2}}\left(k_{x}^{2} x^{2}+k_{y}^{2} y^{2}\right)+\frac{1}{12 k^{2} \rho_{w}^{2}}\left(k_{x}^{4} x^{4}+k_{y}^{4} y^{4}+3 k^{2} k_{x}^{2} x^{2} y^{2}\right) \\
& -\frac{\sin (k s)}{2 k \rho_{w}}\left(p_{x}\left(k_{x}^{2} x^{2}+k_{y}^{2} y^{2}\right)-2 k_{x}^{2} p_{y} x y\right)
\end{aligned}
$$


After averaging the motion over one wiggler period, one obtains the differential equations for particle's transverse motions [6]:

$$
\begin{gathered}
\frac{d^{2} x}{d s^{2}}=-\frac{k_{x}^{2}}{2 k^{2} \rho_{w}^{2}}\left(x+\frac{2}{3} k_{x}^{2} x^{3}+k^{2} x y^{2}\right) \\
\frac{d^{2} y}{d s^{2}}=-\frac{k_{y}^{2}}{2 k^{2} \rho_{w}^{2}}\left(y+\frac{2}{3} k_{y}^{2} y^{3}+\frac{k_{x}^{2} k^{2}}{k_{y}^{2}} x^{2} y\right)
\end{gathered}
$$

Considering the wigglers are built with plane poles, one has $k_{x}=0$.

\section{$3 \quad$ Wiggler as an insertion device in a storage ring}

Now we insert a "wiggler" of only one period (or one cell) into a storage ring located at $s_{w}$. The total Hamiltonian of the ring in the vertical plane can be expressed as follows:

$$
H=H_{0}+\frac{1}{4 \rho^{2}} y^{2}+\frac{k_{y}^{2}}{12 \rho^{2}} y^{4} \lambda_{w} \sum_{i=-\infty}^{\infty} \delta(s-i L)
$$

where $H_{0}$ is the Hamiltonian without the inserted wiggler, $L$ is the circumference of the ring, and $k_{y}=k$. It is obvious that the perturbation is a delta function octupole.

Now, let's recall some useful results obtained in ref. [1] where we have studied analytically the one dimensional dynamic aperture of a storage ring described by the following Hamiltonian:

$$
\begin{aligned}
\mathcal{H}=\frac{p^{2}}{2} & +\frac{K(s)}{2} x^{2}+\frac{1}{3 ! B \rho} \frac{\partial^{2} B_{z}}{\partial x^{2}} x^{3} L \sum_{k=-\infty}^{\infty} \delta(s-k L) \\
& +\frac{1}{4 ! B \rho} \frac{\partial^{3} B_{z}}{\partial x^{3}} x^{4} L \sum_{k=-\infty}^{\infty} \delta(s-k L)+\cdots
\end{aligned}
$$

where

$$
B_{z}=B_{0}\left(1+x b_{1}+x^{2} b_{2}+x^{3} b_{3}+x^{4} b_{4}+\cdots+x^{m-1} b_{m-1}+\cdots\right)
$$


The dynamic aperture corresponding to each multipole is given as:

$$
A_{\text {dyna }, 2 m, x}(s)=\sqrt{2 \beta_{x}(s)}\left(\frac{1}{m \beta_{x}^{m}\left(s_{2 m}\right)}\right)^{\frac{1}{2(m-2)}}\left(\frac{\rho}{\left|b_{m-1}\right| L}\right)^{1 /(m-2)}
$$

where $s_{2 m}$ is the location of the $2 m$ th multipole, $\beta_{x}(s)$ is the beta function in $x$ plane, and $x$ here stands for either horizontal or vertical plane.

Comparing eq. 11 with eq. 12 , by analogy, one finds easily that:

$$
\frac{b_{3}}{\rho} L=\frac{k_{y}^{2} \lambda_{w}}{3 \rho_{w}^{2}}
$$

and the dynamic aperture limited by this one period "wiggler":

$$
A_{1, y}(s)=\frac{\sqrt{\beta_{y}(s)}}{\beta_{y}\left(s_{w}\right)}\left(\frac{3 \rho_{w}^{2}}{k_{y}^{2} \lambda_{w}}\right)^{1 / 2}
$$

where $\beta_{y}(s)$ is the unperturbed beta function. In fact, a wiggler is an insertion device which is composed of a large number of cells, say, $N_{w}$, and the wiggler length $L_{w}=N_{w} \lambda_{w}$. Now, the first question which follows is what the combined effect of these $N_{w}$ cells will be. According to ref. [1], one has:

$$
\frac{1}{A_{N_{w}, y}^{2}(s)}=\sum_{i=1}^{N_{w}} \frac{1}{A_{i, y}^{2}}=\sum_{i=1}^{N_{w}}\left(\frac{k_{y}^{2}}{3 \rho_{w}^{2} \beta_{y}(s)}\right) \beta_{y}^{2}\left(s_{i, w}\right) \frac{L_{w}}{N_{w}}
$$

where the index $i$ indicates different cell. When $N_{w}$ is a large number, Eq. 17 can be simplified as:

$$
\frac{1}{A_{N_{w}, y}^{2}(s)}=\frac{k_{y}^{2}}{3 \rho_{w}^{2} \beta_{y}(s)} \int_{s_{w_{0}}-L_{w} / 2}^{s_{w_{0}}+L_{w} / 2} \beta_{y}^{2}(s) d s
$$

where $s_{w_{0}}$ correspond to the center of the wiggler. If the variation of the unperturbed beta function inside the wiggler is approximated as linear, one gets

$$
A_{N_{w}, y}(s)=3 \sqrt{\frac{\beta_{y}(s)\left(\beta_{y, 2}-\beta_{y, 1}\right)}{\beta_{y, 2}^{3}-\beta_{y, 1}^{3}}} \frac{\rho_{w}}{k_{y} \sqrt{L_{w}}}
$$

where $\beta_{y, 1}$ and $\beta_{y, 2}$ correspond to the beta function values at the two extremities of the wiggler. As is well known, the inserted wiggler perturbs linear 
optics also, such as tune shifts and beta functions. In our specific case [7], we have $\Delta \nu_{x}=0, \Delta \beta_{x}=0$, and

$$
\begin{gathered}
\Delta \nu_{y} \approx \frac{L_{w} \beta_{a v, y}}{8 \pi \rho_{w}^{2}} \\
\frac{\Delta \beta_{y}}{\beta_{y}} \approx-\frac{L_{w} \beta_{a v, y} \cos \left(2 \nu_{y}\left(\pi-\left|\phi-\phi_{w}\right|\right)\right)}{4 \rho_{w}^{2} \sin \left(2 \pi \nu_{y}\right)}
\end{gathered}
$$

or

$$
\left(\frac{\Delta \beta_{y}}{\beta_{y}}\right)_{\max } \approx\left|2 \pi \Delta \nu_{y} / \sin \left(2 \pi \nu_{y}\right)\right|
$$

where $\beta_{a v, y}$ is the averaged beta function within the wiggler. The fact that the tune shift and the beta function insite the wiggler vary in a complex way makes us assume that the octupole like cells of the wiggler are independent from one to another, and permits us to arrive at the expression in eq. 17.

The second question which follows is how about the total dynamic aperture of the storage ring including many wigglers and other nonlinear components. Assuming that the dynamic aperture of the ring without the wigglers' effects is $A_{y}$ and that there are $M$ wigglers to be inserted inside the ring at different places, one has the total dynamic aperture expressed as:

$$
A_{\text {total }, y}(s)=\frac{1}{\sqrt{\frac{1}{A_{y}(s)^{2}}+\sum_{j=1}^{M} \frac{1}{A_{j, w, y}(s)^{2}}}}
$$

where $A_{j, w, y}$ denotes the dynamic aperture limited by the $j$ th wiggler.

\section{$4 \quad$ Numerical examples}

Now we take TESLA damping ring for example with permanent magnet wigglers [2], where one has $E_{0}=5 \mathrm{GeV}, B_{0}=1.68 \mathrm{~T}, \lambda_{w}=0.4 \mathrm{~m}, N_{w}=$ $12, \beta_{y, 1}=9 \mathrm{~m}, \beta_{y, 2}=15 \mathrm{~m}$, and total wiggler number $M=45$. Without considering the dynamic aperture limited by other nonlinear components, by applying eqs. 19 and 23, one finds that $A_{t o t a l, y}\left(s_{w_{0}}\right)=21 \mathrm{~mm}$. Recalling the gap of the wiggler [2], $g=25 \mathrm{~mm}$, one concludes that the parameter choice for the wigglers in TESLA damping is roughly reasonable. 


\section{Conclusion}

In this paper we have developed the analytical dynamic aperture formulae limited by wigglers in storage rings, which are very efficient and powerful in designing and operating damping rings and synchrotron radiation facilities.

\section{Acknowledgements}

The author thanks T. Garvey and P. Bambade for their encouragements.

\section{References}

[1] J. Gao, "Analytical estimation of the dynamic apertures of circular accelerators", Nucl Instr. and Methods, A451 (3) (2000), p. 545.

[2] W. Decking, "Optical layout of the TESLA $5 \mathrm{GeV}$ damping ring", TESLA report 2001-11.

[3] A. Ropert, "Lattice and emittances", CERN 98-04, p. 91.

[4] T. Lee, et al., "Analysis of the effects superconducting wiggler on beam dynamics in storage rings", Proceedings of EPAC2000, Vienna, Austria, p. 1030 .

[5] J.M. Jowett, "Emittance control with wigglers", LEP Note 389, 1982.

[6] A. Fedorova, et al., "Nonlinear beam dynamics and effects of wigglers", Proceedings of EPAC2000, Vienna, Austria, p. 2325.

[7] M. Katoh and Y. Kamiya, "Effect of insertion devices on beam parameters", Proceedings of PAC87, p. 437. 